ARTICLE

DOI: $10.1038 / s 41467-018-04996-x$

\title{
Evolutionary trade-offs associated with loss of PmrB function in host-adapted Pseudomonas aeruginosa
}

Laura Bricio-Moreno1, Victoria H. Sheridan', Ian Goodhead², Stuart Armstrong1,3, Janet K.L. Wong1, Elaine M. Waters ${ }^{1,6}$, Joscelyn Sarsby ${ }^{4}$, Stavros Panagiotou' ${ }^{1}$ James Dunn ${ }^{1}$, Adrita Chakraborty ${ }^{1}$, Yongliang Fang ${ }^{5}$, Karl E. Griswold (10 ${ }^{5}$, Craig Winstanley ${ }^{1}$, Joanne L. Fothergill ${ }^{1}$, Aras Kadioglu ${ }^{1} \&$ Daniel R. Neill (i) ${ }^{1}$

Pseudomonas aeruginosa colonises the upper airway of cystic fibrosis (CF) patients, providing a reservoir of host-adapted genotypes that subsequently establish chronic lung infection. We previously experimentally-evolved $P$. aeruginosa in a murine model of respiratory tract infection and observed early-acquired mutations in $\mathrm{pmr} B$, encoding the sensor kinase of a two-component system that promoted establishment and persistence of infection. Here, using proteomics, we show downregulation of proteins involved in LPS biosynthesis, antimicrobial resistance and phenazine production in pmrB mutants, and upregulation of proteins involved in adherence, lysozyme resistance and inhibition of the chloride ion channel CFTR, relative to wild-type strain LESB65. Accordingly, pmrB mutants are susceptible to antibiotic treatment but show enhanced adherence to airway epithelial cells, resistance to lysozyme treatment, and downregulate host CFTR expression. We propose that $P$. aeruginosa pmrB mutations in CF patients are subject to an evolutionary trade-off, leading to enhanced colonisation potential, CFTR inhibition, and resistance to host defences, but also to increased susceptibility to antibiotics.

\footnotetext{
${ }^{1}$ Institute of Infection and Global Health, University of Liverpool, Liverpool L69 7BE, UK. ${ }^{2}$ School of Environment and Life Sciences, University of Salford, Salford M5 4WT, UK. ${ }^{3}$ NIHR Health Protection Research Unit in Emerging and Zoonotic Infections, University of Liverpool, Liverpool L69 3GL, UK. ${ }^{4}$ Institute of Integrative Biology, University of Liverpool, Liverpool L69 7ZB, UK. ${ }^{5}$ Thayer School of Engineering, Dartmouth, Hanover, NH 03755, USA. ${ }^{6}$ Present address: Department of Microbiology, School of Natural Science, National University of Ireland, Galway H91 TK33, Ireland. These authors contributed equally: Joanne L. Fothergill, Aras Kadioglu, Daniel R. Neill Correspondence and requests for materials should be addressed to

J.L.F. (email: j.fothergill@liverpool.ac.uk) or to D.R.N. (email: d.neill@liverpool.ac.uk)
} 
P seudomonas aeruginosa lung infections are the single biggest cause of mortality in people with cystic fibrosis $(\mathrm{CF})^{1,2}$. Such infections are typically chronic, highly resistant to antimicrobial therapy and punctuated by periodic exacerbations. $P$. aeruginosa is ubiquitous in the environment and will thrive under diverse conditions ${ }^{3}$. Such adaptability is facilitated by a large and plastic genome ${ }^{4}$. However, we still know relatively little about the early adaptation events that take place within the host during infection.

Previous work by ourselves and others has suggested that the upper airways (the paranasal sinuses and nasopharynx) may function as a protected niche and "evolutionary nest" for $P$. aeruginosa, allowing prolonged colonisation in an environment with fewer immune defence agents and greater nutrient availability than the lung ${ }^{5-8}$. Gradual adaptation to the host environment paves the way for subsequent seeding into the lungs and establishment of chronic infection. When $P$. aeruginosa was cultured simultaneously from the sinuses and lungs of patients who had undergone Endoscopic Sinus Surgery, isolates from the two sites were genetically identical in $95 \%$ of patients ${ }^{8}$. Furthermore, $P$. aeruginosa form biofilms in the sinuses without triggering strong cellular inflammatory responses ${ }^{8}$.

We have previously used a chronic $P$. aeruginosa respiratory infection model in mice to demonstrate that, whilst host immune clearance mechanisms efficiently remove bacteria infecting the lung, colonisation persists in the nasopharynx for long periods ${ }^{6}$. The same study showed that prolonged colonisation is associated with subsequent reseeding events, whereby $P$. aeruginosa reappear in the lungs several weeks after initial immune clearance. Genome sequencing of isolates taken from nasopharynx and lungs one month post-infection revealed the presence of a single nucleotide polymorphism (SNP) resulting in a missense mutation in $p m r B$. This mutation was present in multiple isolates taken from different mice ${ }^{6}$. We have since compared the PmrB amino acid sequences of the Liverpool Epidemic Strain (LES)B65 used for the initial infection (DDBJ sequence read archive ERS2269679 (http://ddbj.nig.ac.jp/DRASearch/sample?acc=ERS2269679)) and PAO1 (GenBank AAG08163 (https://www.ncbi.nlm.nih.gov/ protein/AAG08163.1)) and observed no differences, suggesting the SNP acquired by LESB65 in the mouse model was not a reversion mutation. An isolate with this $\operatorname{pmrB}$ SNP showed a greatly enhanced ability to colonise mouse lungs compared to both the LESB65 used in the original infection and an isolate taken from the mouse model that did not have a $p m r B$ SNP (referred to as $p m r B$ WT hereafter) ${ }^{6}$. Thus, our previous study suggested that acquisition of $p m r B$ mutations early in infection appeared to confer a selective advantage in the establishment of lung infection. The Liverpool Epidemic Strain is a widely studied, transmissible lineage of $P$. aeruginosa from CF patients ${ }^{9}$. Isolates of this strain have been shown to have a modified LPS structure, lacking an $\mathrm{O}$-antigen ${ }^{9,10}$, similar to other clinical isolates. Here, we describe the phenotypic changes associated with $p m r B$ mutation that enable efficient lung colonisation.

Two-component regulatory systems are key regulators of virulence in bacteria, allowing the expression of multiple genes to be coupled to changes in the environment. P. aeruginosa has multiple two-component systems, amongst which PhoPQ and GacAS are probably the best characterised ${ }^{11}$. Phenotypic changes in $P$. aeruginosa that characterise the switch from acute to chronic infection are under the control of GacAS ${ }^{12,13}$. PmrAB is another such two-component system, involved in the modification of LPS in P. aeruginosa, and mutations in the pmrAB operon have been linked to resistance to cationic antimicrobial peptides $^{14}$.

The mouse infection model from which we previously isolated $P$. aeruginosa $p m r B$ SNP mutants did not include any antibiotic treatment ${ }^{6}$ and so we hypothesised that the selective pressure for mutation may come from host-derived antimicrobial peptides or that the phenotype conferred by the SNP mutation might be different from those previously reported. This was the starting point for the present study, wherein we describe a significantly altered phenotype of $P$. aeruginosa lacking $p m r B$ or those carrying the in vivo acquired $p m r B$ SNP and demonstrate that these changes aid in multiple facets of host colonisation by conferring resistance to host antimicrobials, enhanced adherence to host surfaces and an ability to modulate the local environment in a way that drives a $\mathrm{CF}$ lung phenotype. Our findings highlight the key role played by the $P$. aeruginosa PmrAB two-component regulatory system in regulating a wide range of biological processes and virulence mechanisms. Finally, we propose that the evolutionary trade-off associated with $p m r B$ mutation may offer opportunities for therapeutic intervention, as enhanced in vivo fitness is offset by increased susceptibility to several classes of antibiotics.

\section{Results}

Loss-of-function $p m r B$ mutations confer lysozyme resistance. Neutrophils are a major defence against bacterial infection of the respiratory tract and neutrophil degranulation releases antimicrobial peptides and enzymes ${ }^{15}$. In order to determine the effect of $p m r B$ mutation on susceptibility to neutrophil killing, human HL-60 neutrophils and mouse neutrophils isolated from bone marrow were cultured with phorbol myrisate acetate (PMA) to induce degranulation and culture supernatants (containing granule contents) were transferred to LESB65, a $p m r B$ deletion mutant on the LESB65 background $(\Delta p m r \mathrm{~B})$ or two LESB65-derived isolates taken from 28 days post-infection in the mouse infection model, one with and one without a $p m r B$ SNP (referred to as $p m r B$ SNP and $p m r B$ WT, respectively, hereafter, and as NP22_2 and NP22_4 in ref. $^{6}$ ). A full description of the additional mutations in the LESB65-derived isolates, determined by PacBio sequencing, can be found in Supplementary Table 1. Isolates with mutated $p m r B$ ( $p m r B$ deletion and SNP mutants) showed significantly enhanced resistance to killing by neutrophil-conditioned media (Fig. 1a, b). To determine whether resistance to a neutrophil-derived antimicrobial accounted for this phenotype, the four bacterial isolates were cultured with $\beta$-defensin 2, mCRAMP (the murine homologue of the human cathelicidin LL-37) or lysozyme. The two pmrB mutants showed significantly enhanced resistance to lysozyme killing (Fig. 1c), but not to $\beta$-defensin 2 or mCRAMP in two-way ANOVA analysis (Supplementary Fig. 1A and B). Lysozyme is abundant in the mammalian airway ${ }^{16}$ and is active against both Gram-positive and Gram-negative species ${ }^{17,18}$. Significant differences in resistance between isolates were observed at lysozyme concentrations within the physiological range found in saliva ${ }^{19}$. Lysozyme resistance obtained through $p m r B$ mutation may, therefore, provide a selective advantage in the establishment of respiratory tract infection.

Antimicrobial susceptibility in pmrB mutants. Mutations in the pmrAB system have previously been linked to changes in resistance to antibiotics ${ }^{14,20}$. To determine whether the phenotypic changes in pmrB mutants that conferred lysozyme resistance also influenced resistance to antibiotics, minimum inhibitory concentration (MIC) assays were performed (Table 1, Supplementary Fig. 2A-E). Strikingly, isolates with $p m r B$ mutations were notably more susceptible to 6 of the 7 antibiotics tested, when antibiotic concentration required to inhibit $90 \%$ growth (MIC90) was determined (Table 1). These antibiotics included tobramycin and colistin, commonly used in the treatment of $P$. aeruginosa infections in people with CF. Similarly, $P$. aeruginosa with a transposon insertion in $p m r B$ showed enhanced susceptibility to both tobramycin and 
a
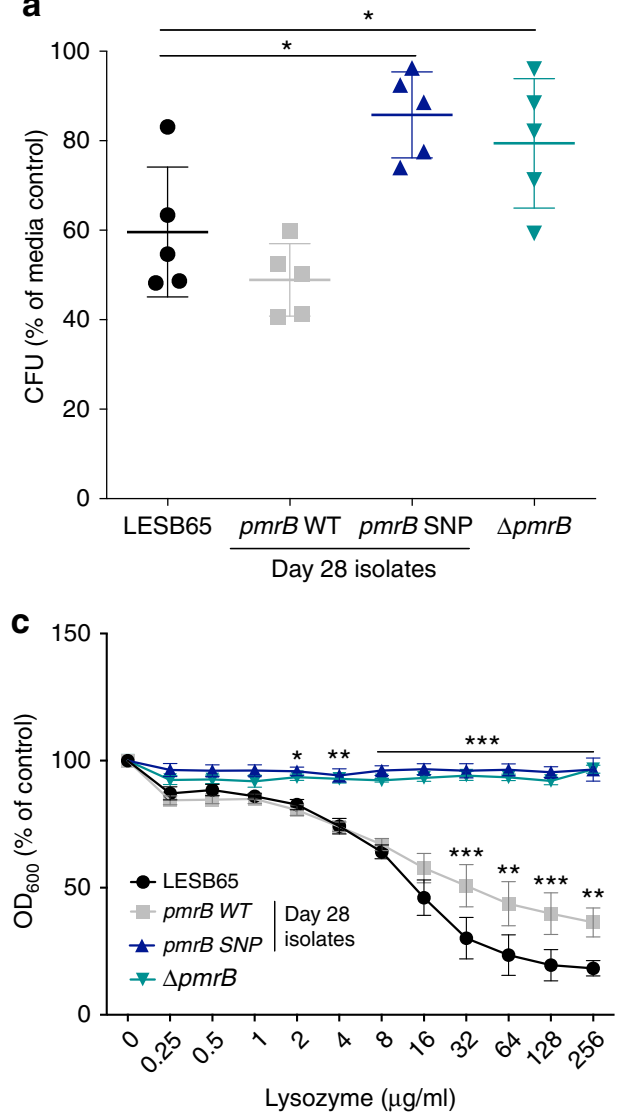

b

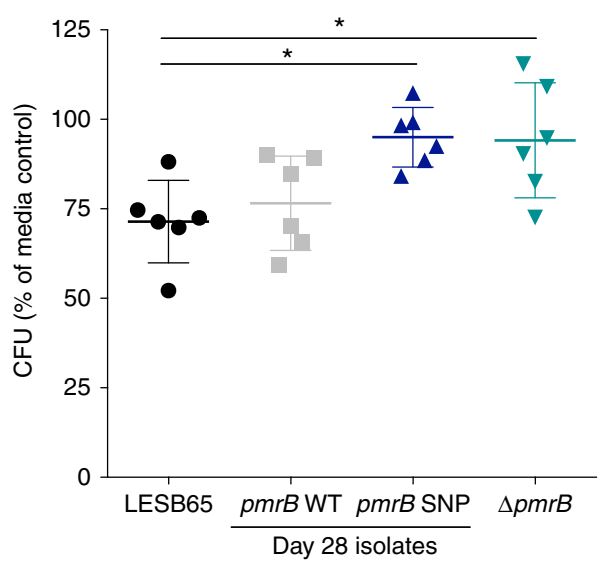

Fig. 1 P. aeruginosa pmrB mutants are resistant to host defence molecules and lysozyme killing. Supernatants from phorbol 12-myristate 13-acetate (PMA)stimulated (a) HL-60 or (b) mouse bone marrow neutrophil cultures were transferred to mid-log growth $P$. aeruginosa. Colony forming units (CFU) as a percentage of control. Control bacteria were exposed to supernatant from non-activated (no PMA) HL-60 cultures. c Optical density at 600 nm of $P$. aeruginosa grown in the presence of lysozyme (mean \pm s.d.). Results are expressed as a percentage of the no-lysozyme control for each isolate and are a composite of 3 biological replicates per isolate, each containing 3 technical replicates. Asterisks $\left({ }^{\star}\right)$ represent significant differences in ANOVA performed on CFU counts $(\mathbf{a}, \mathbf{b})$ or OD readings, with lysozyme as a covariant (c), with Dunnett's multiple comparison test vs. LESB65. ${ }^{\star} p<0.05,{ }^{\star \star} p<0.01,{ }^{\star \star \star} p<0.005$

colistin, relative to the parental PAO1 strain (Supplementary Table 2). Thus, on both the LES and PAO1 background, loss of $p m r B$ function is associated with a relative increase in antibiotic susceptibility. The evolution of resistance to lysozyme at the cost of antibiotic susceptibility is an example of collateral sensitivity, as has been previously described for $P$. aeruginosa pmrB mutants, in which resistance to gentamicin was associated with penicillin sensitivity ${ }^{21}$.

Chronic $P$. aeruginosa lung infections are characterised by bacterial growth in biofilm that provides resistance to antibiotic treatment and host immune responses ${ }^{22,23}$. Although differences in tobramycin sensitivity between isolates were less pronounced when grown in an artificial sputum model (ASM) of biofilm growth, the increased susceptibility of $p m r B$ mutants relative to LESB65 was evident at 4 and $8 \mu \mathrm{g} / \mathrm{ml}$ tobramycin (Supplementary Fig. 3A).

The effectiveness of lysozyme is also reduced in biofilms and in the chronically infected lung, due to electrostatic sequestration of the enzyme by infection-associated anionic biopolymers ${ }^{24,25}$. This was clear when LESB65 was grown in ASM, where it showed complete resistance to lysozyme at all concentrations tested (data not shown). However, when electrostatic sequestration by anionic biopolymers in ASM was negated through use of chargeengineered lysozyme ${ }^{24,26}$, the differential lysozyme resistance of pmrB mutants could still be observed (Supplementary Fig. 3B). This charge-engineering did not significantly enhance lysozyme killing of any of the four isolates when experiments were performed in planktonic culture (Supplementary Fig. 4).
Proteomics analysis of $p m r B$ mutants. The dichotomous phenotypic changes in $p m r B$ mutants, with enhanced resistance to a key host derived antimicrobial but increased susceptibility to antibiotics, suggested that $p m r B$ mutation might lead to significant changes in bacterial gene expression, protein production or physiology. To explore this, we performed proteomic analysis of LESB65 and $\triangle p m r B$ grown in liquid culture. We found 216 proteins with significantly different abundance in LESB65 cultures compared to $\triangle p m r B$ (Fig. 2, Supplementary Data 1 and Supplementary Fig. 5), and these included the two proteins of the PmrAB two-component regulatory system, as well as key virulence factors (Table 2). Of the proteins identified as differentially abundant between the two isolates, $60 \%(129 / 216)$ were enriched in $\triangle p m r B$.

Supportive of our phenotypic observations, several proteins with described roles in antimicrobial resistance were significantly more abundant in LESB65 than in $\triangle p m r B$. These included the protein products of several genes of the arn locus, which is crucial to the synthesis of the lipid A component of bacterial lipopolysaccharide and which mediates resistance to cationic antimicrobials through addition of 4-amino-L-arabinose to lipid $\mathrm{A}^{14}$. Expression of the arn locus has been shown to be under the control of two-component systems including PhoPQ and PmrAB ${ }^{14,27,28}$. Using MALDI-TOF mass spectrometry, differences in lipid A traces were detected. Peaks corresponding to both penta-acyl lipid A and hexa-acyl lipid A were significantly reduced in $\triangle p m r B$ compared to the isogenic 
Table 1 Antibiotic susceptibility of Psuedomonas aeruginosa isolates

Mouse model isolates

LESB65 pmrBWT pmrB SNP $\quad \Delta p m r B$

MIC50

\begin{tabular}{|c|c|c|c|c|}
\hline Chloramphenicol & $2(2-8)$ & $8(4-16)$ & $8(4-16)$ & $4(4-16)$ \\
\hline Ciprofloxacin & $1(1)$ & $0.5(0.5-1)$ & $0.5(0.25-0.5)$ & $0.25(0.25-1)$ \\
\hline Colistin & $2(2-4)$ & $4(0.25-8)$ & $0.25(0.25-1)$ & $0.25(0.25-1)$ \\
\hline Gentamicin & $>128(128->128)$ & $128(128)$ & $16(16)$ & $16(16)$ \\
\hline Polymyxin B & $0.5(0.25-1)$ & $0.5(0.25-0.5)$ & $0.25(0.25-0.5)$ & $0.25(0.25)$ \\
\hline Tobramycin & $32(4-32)$ & $16(16-32)$ & $2(2-4)$ & $2(1-4)$ \\
\hline Tetracycline & $8(8-16)$ & $16(8-32)$ & $4(4-8)$ & $4(0.5-8)$ \\
\hline
\end{tabular}

MIC90

\begin{tabular}{|c|c|c|c|c|}
\hline Chloramphenicol & $>128(>128)$ & $128(64->128)$ & $>128(128->128)$ & $>128(128->128)$ \\
\hline Ciprofloxacin & $8(2-16)$ & $2(2)$ & $1(0.5-1)$ & $1(1)$ \\
\hline Colistin & $>128(32->128)$ & $128(4->128)$ & $1(1-8)$ & $1(0.25-2)$ \\
\hline Gentamicin & $>128(>128)$ & $>128(>128)$ & $128(64-128)$ & $128(128)$ \\
\hline Polymyxin B & $16(8-64)$ & $16(16-128)$ & $0.5(0.25-0.5)$ & $0.5(0.25-1)$ \\
\hline Tobramycin & $>128(16->128)$ & $>128(>128)$ & $16(16-128)$ & $32(16-128)$ \\
\hline Tetracycline & $>128(>128)$ & $>128(>128)$ & $128(128->128)$ & $128(128->128)$ \\
\hline
\end{tabular}

Minimum inhibitory concentration ( $\mu \mathrm{g}$ per $\mathrm{ml}$ ) required for $50 \%$ or $90 \%$ growth inhibition. Median MIC50 and MIC 90 from a minimum of 5 replicates (range 5 - 9 ) is in bold font, range is in brackets. The two/three most sensitive isolates for each antibiotic are shaded grey

wild type LESB65 (Supplementary Fig. 6). Thus $\Delta p m r B$, that demonstrated reduced expression of proteins of the arn locus, may also have reduced lipid A content.

Protein PA4775 (T224_RS56950 in LESB65), which was enriched in LESB65 but not $\triangle p m r B$ has also been linked to resistance to both antibiotics and oxidative damage via an LPSdependent mechanism ${ }^{29}$. Other proteins with roles in antimicrobial resistance that were more abundant in LESB65 include CprA, which contributes to polymyxin resistance by an arn-independent pathway $^{30}$, and the multidrug efflux pump protein $\mathrm{MexE}^{31}$.
Proteins involved in phenazine biosynthesis were also enriched in LESB65 as compared to $\triangle p m r B$. Quantification of pyocyanin in cultures of LESB65, $p m r B$ WT, $p m r B$ SNP and $\Delta p m r B$, confirmed that loss of $\mathrm{PmrB}$ function is associated with reduced pyocyanin production (Supplementary Fig. 7). Pyocyanin is a key virulence factor of $P$. aeruginosa, and it is unclear how its downregulation in $\triangle p m r B$ might contribute to increased survival in vivo. Notably however, pyocyanin is a stimulator of IL-8 release from the human airway epithelium and reduced production may aid evasion of neutrophil responses prior to establishment of biofilm ${ }^{32,33}$. 
The lysozyme resistance observed with $\Delta p m r B$ is likely a consequence of increased production of the inhibitor of vertebrate

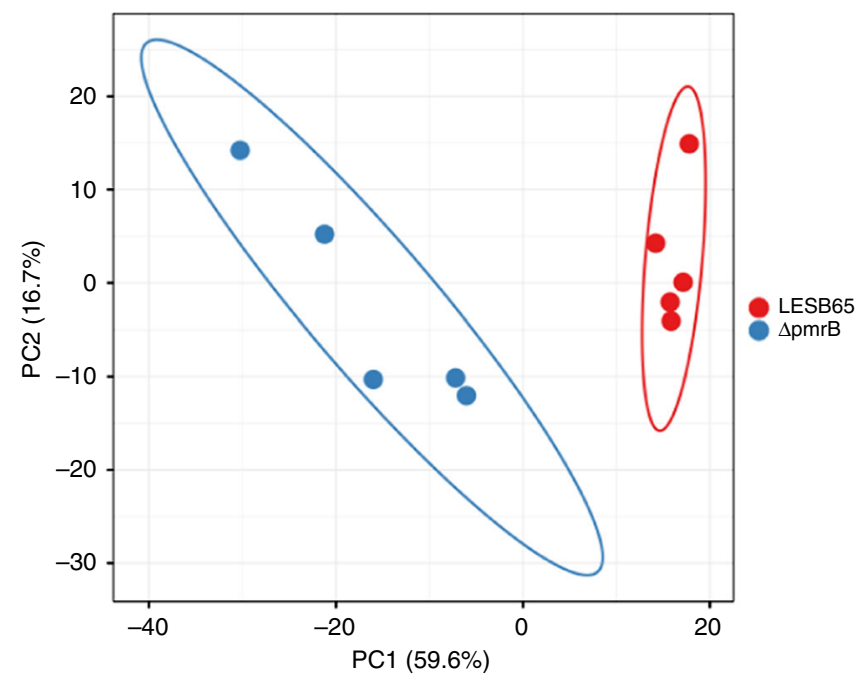

Fig. 2 Principal component biplot of LESB65 and $\Delta p m r B$ proteomics data showing proteins with significantly different abundance between samples. Five biological replicates were performed for each isolate. Unit variance scaling is applied to rows; SVD with imputation is used to calculate principal components. $X$ axis and $Y$ axis show principal component 1 and principal component 2 that explain $59.6 \%$ and $16.7 \%$ of the total variance, respectively. Prediction ellipses are such that with probability 0.95 , a new observation from the same group will fall inside the ellipse. PCA analysis of the full dataset, with all detected proteins, can be found in Supplementary Fig. 4 lysozyme (Ivy) protein, enriched 2.66-fold in $\triangle p m r B$ relative to LESB65 (Table 2). An increase in cif expression was also detected in $\triangle p m r B$ and $p m r B$ SNP using qPCR (Supplementary Table 3). Ivy is a proteinaceous inhibitor of lysozyme first described in Escherichia coli ${ }^{34}$. E. coli ivy knockout mutants exhibit growth defects in lysozyme-rich environments, including hen egg white and saliva ${ }^{35}$. However, Deckers et al. found no effect of ivy deletion in $P$. aeruginosa $\mathrm{PAO} 1$ on growth in lysozyme-containing saliva or egg white, perhaps due to compensatory lysozyme resistance mechanisms such as that mediated by $\mathrm{MliC}^{36}$.

Modulation of CFTR by pmrB mutants. The cystic fibrosis transmembrane conductance regulator (CFTR) inhibitory protein Cif was 5.65 -fold more abundant in $\triangle p m r B$ than in LESB65 (Table 2). An increase in cif expression was also detected in $\triangle p m r B$ and $p m r B$ SNP using qPCR (Supplementary Table 3). Cif inhibits endocytic recycling of CFTR in human epithelial cells $^{37}$, decreasing apical membrane expression of the protein ${ }^{38}$. Furthermore, Cif is expressed in the CF lung ${ }^{38}$, suggesting that $P$. aeruginosa can phenocopy the CF lung environment, potentially facilitating colonisation and chronic infection. To determine whether the high levels of Cif measured in $\triangle p m r B$ samples was of functional relevance, LESB65 and $\triangle p m r B$ were cultured with A549 human pulmonary epithelial cells and incubated overnight before CFTR expression was assessed by flow cytometry. Strikingly, although A549 CFTR expression was unaffected by co-culture with LESB65, significantly decreased CFTR expression was evident on A549 cells cultured with $\triangle p m r B$ (Fig. 3a, b). Similarly, $p m r B$ SNP, but not $p m r B$ WT was able to reduce CFTR expression on A549 cells (Fig. 3b) and the same phenotypes were observed when Detroit human pharyngeal epithelial cells were used (Fig. 3c). This raises the possibility that

Table 2 Differential protein abundance in LESB65 vs. $\Delta$ pmrB grown in liquid culture

\begin{tabular}{|c|c|c|c|c|}
\hline Biological process & Protein name & Gene & $\begin{array}{l}\text { Fold } \\
\text { change }\end{array}$ & $q$-value \\
\hline \multirow[t]{3}{*}{ Type IV pili } & Type IV fimbrial biogenesis protein PilV & pilV PA4551 & 6.14 & 0.003560888 \\
\hline & Type IV fimbrial biogenesis protein PilY2 & pilY2 PA4555 & 5.80 & 0.018144003 \\
\hline & Pilus associated adhesin PilY1 & pilY1 PA4554 & 2.33 & 0.026743646 \\
\hline CFTR inhibition & CFTR inhibitory factor CIF & cif PA2934 & 5.65 & 0.014178322 \\
\hline Lysozyme inhibition & Inhibitor of vertebrate lysozyme & ivy PA3902 & 2.66 & 0.049597254 \\
\hline Two-component & PmrB: two-component signal sensor kinase & pmrB PA4777 & -20.13 & 0.000337869 \\
\hline signalling & PmrA: two-component signal response regulator & pmrA PA4776 & -25.95 & 0.000615523 \\
\hline \multirow[t]{7}{*}{ Phenazine biosynthesis } & Phenazine biosynthesis protein PhzD & $\begin{array}{l}\text { phzD1 phzD2 PA1902 } \\
\text { PA4213 }\end{array}$ & -5.37 & 0.001208844 \\
\hline & Phenazine biosynthesis protein PhzE & $\begin{array}{l}\text { phzE1 phzE2 PA1903 } \\
\text { PA4214 }\end{array}$ & -3.93 & 0.001208844 \\
\hline & Pyridoxal 5'-phosphate synthase & phzG1 PA4216 & -2.98 & 0.01260054 \\
\hline & Phospho-2-dehydro-3-deoxyheptonate aldolase & $\begin{array}{l}\text { phzC1 phzC2 PA1901 } \\
\text { PA4212 }\end{array}$ & -6.89 & 0.000837624 \\
\hline & RND multidrug efflux membrane fusion protein MexE* & MexE PA2493 & -5.39 & 0.029669765 \\
\hline & Anthranilate--CoA ligase & pqsA PA0996 & -2.82 & 0.040457292 \\
\hline & BfmS & bfmS PA4102 & -3.79 & 0.020754134 \\
\hline \multirow[t]{5}{*}{ LPS modification } & $\begin{array}{l}\text { 4-deoxy-4-formamido-L-arabinose-phosphoundecaprenol deformylase } \\
\text { ArnD }\end{array}$ & arnD PA3555 & -26.31 & 0.000200769 \\
\hline & Bifunctional polymyxin resistance protein ArnA & $\operatorname{arnA~PA3554~}$ & -18.63 & 0.000337869 \\
\hline & $\begin{array}{l}\text { Undecaprenyl phosphate-alpha-4-amino-4-deoxy-L-arabinose arabinosyl } \\
\text { transferase }\end{array}$ & arnT PA3556 & -11.37 & 0.003786234 \\
\hline & UDP-4-amino-4-deoxy-L-arabinose--oxoglutarate aminotransferase & arnB PA3552 & -7.17 & 0.01284628 \\
\hline & 3-deoxy-D-manno-octulosonic-acid (KDO) transferase & waaA PA4988 & -3.85 & 0.040439226 \\
\hline \multirow{3}{*}{$\begin{array}{l}\text { Antimicrobial } \\
\text { resistance }\end{array}$} & CprA & cprA PA1559 & -19.96 & 0.005901835 \\
\hline & CprA & cprA PA1560 & -30.39 & 2.78965E-05 \\
\hline & Uncharacterised protein & PA4775 & -22.89 & 0.00018425 \\
\hline
\end{tabular}

Proteins dowregulated in $\triangle p m r B$ relative to LESB65 are denoted with a minus fold-change

${ }^{*}$ MexE is also involved in antibiotic resistance 
the success of $p m r B$ mutants in vivo may reflect an ability to modulate the host environment to create conditions conducive to bacterial growth.

Loss of cif or ivy attenuates colonisation potential. To determine whether Ivy and Cif make contributions to P. aeruginosa colonisation and survival in vivo, PAO1 transposon mutants with insertions in ivy or cif were compared to the parental PAO1 strain in a mouse inhalation infection model ${ }^{6}$. Lack of either Cif or Ivy led to a significant defect in establishment of infection that was more pronounced in lung than nasopharynx (Fig. 4a, b). Cif and Ivy mutant lung CFU were significantly reduced vs. PAO1 at days 1, 3 and 7 post-infection and infection was cleared from lung in 5/6 Cif and Ivy-infected mice, but only 2/6 PAO1infected mice (Fig. 4a).

Enhanced airway adhesion and motility in $p m r B$ mutants. Several proteins involved in type IV pili biosynthesis were significantly more abundant in $\triangle p m r B$ than in LESB65. Type IV pili play crucial roles in mediating adhesion to host surfaces and in bacterial motility, but also in environmental sensing and virulence ${ }^{39}$. To determine whether increased abundance of type IV pili proteins in $\triangle p m r B$ enabled more efficient adherence to airway epithelial cells, adhesion/invasion assays were performed with A549 human alveolar epithelial cells and Detroit human pharyngeal epithelial cells (Fig. 5a, b). In all samples tested, less than $15 \%$ of bacteria were found to have adhered to epithelial cells after $2 \mathrm{~h}$ of co-culture, but the proportion of adhered bacteria was significantly higher in $\triangle p m r B$-epithelial cell cocultures than in LESB65-epithelial cell cultures (Fig. 5a). Adherence is key to persistence and establishment of biofilms ${ }^{40}$, and increased adherence in $p m r B$ mutants may contribute to in vivo virulence.

The proportion of invaded bacteria found to be residing within A549 epithelial cells following triton X-induced lysis was higher for LESB65 than for $\triangle p m r B$ (Fig. $5 \mathrm{~b}, p=0.07$ in unpaired $t$-test with Welch's correction when considered as a percentage of total CFU, $p<0.01$ when considered as a percentage of adhered CFU only). This may be a consequence of the CFTR downregulation induced by $\triangle p m r B$, as CFTR has been implicated in $P$. aeruginosa cellular invasion ${ }^{41-43}$. No difference between LESB65 and $\triangle$ pmrB was observed for invasion of Detroit pharyngeal epithelial cells (Fig. 5b) that express lower levels of CFTR (Fig. 3c).

Motility in $P$. aeruginosa is mediated via both type IV pili and flagella $^{44}$. Several pili proteins were more abundant in $\Delta p m r B$ than in LESB65 in the proteomic analysis. In motility assays, both $\triangle p m r B$ and $p m r B$ SNP showed enhanced flagella-dependent and type IV pili-dependent swarming ${ }^{45}$ relative to LESB65 or $p m r B$ WT (Fig. 5c, d). No difference in flagella-dependent swimming or pili-dependent twitching was observed (Supplementary Fig. 8A-D). Swarming takes place on the sort of viscous or semisolid surfaces typified by the mucous of the CF lung and swarming motility is thought to be linked to the expression of virulence factors ${ }^{46}$. Thus, enhanced swarming in $\triangle p m r B$ may be an adaptation to the host environment.

Proteomics comparison of $p m r B$ SNP with $p m r B$ deletion mutant. Our in vitro data suggested that deletion of $p m r B$ reproduced the phenotype of the mouse model pmrB SNP isolate. Direct proteomic comparison of LESB65, $p m r B$ SNP and $\Delta p m r B$ confirmed this, with the two $p m r B$ mutant strains clustering together, and away from LESB65 in principal component analysis (Fig. 6). PmrA $\left(p=2.76 \times 10^{-5}\right)$ and $\operatorname{PmrB}\left(p=5.74 \times 10^{-10}\right)$ were significantly less abundant in $p m r B$ SNP relative to LESB65, a

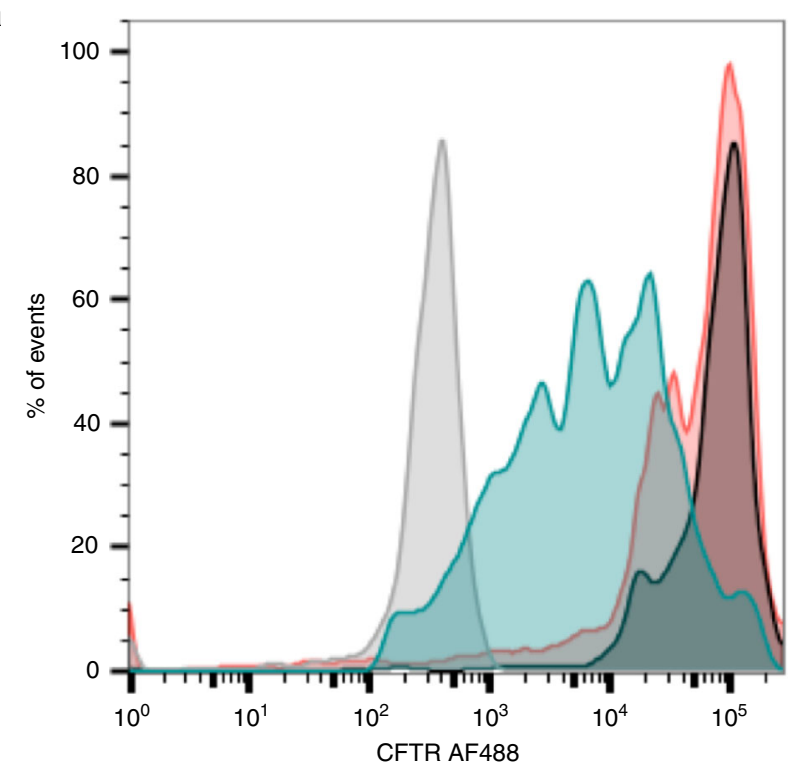

b

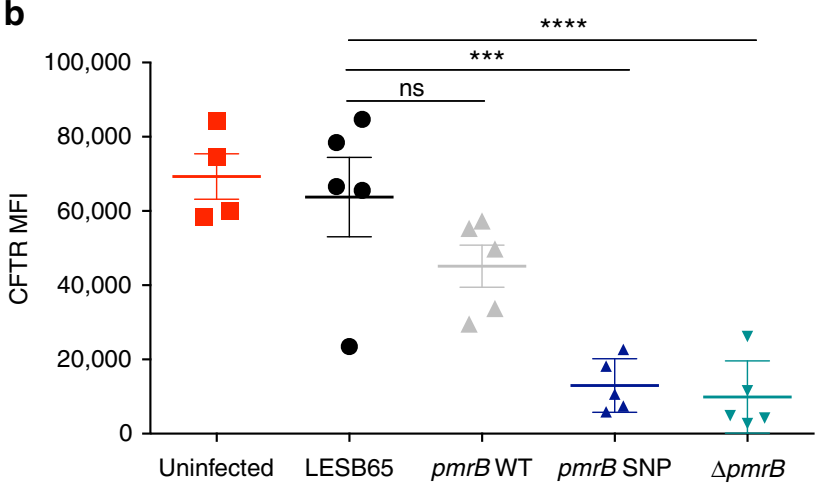

C

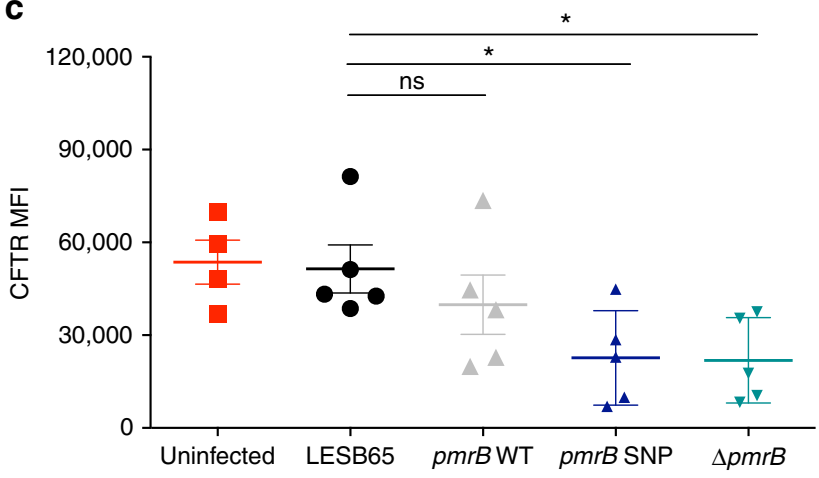

Fig. $3 \Delta p m r B$ downregulates CFTR expression on airway epithelial cells. a CFTR expression on uninfected (red), LESB65 infected (black) and $\Delta p m r B$ infected (blue) A549 airway epithelial cells as measured by flow cytometry and compared to isotype control staining (grey). CFTR alexa fluor 488 median fluorescent intensity (MFI) on stained (b) A549 and (c) Detroit cells. Data in (b) are a composite of two independent experiments with two-three biological replicates per condition in each experiment. Data in (c) are from a single experiment containing 4 biological replicates.

Asterisks $\left(^{*}\right)$ represent significant differences in one-way ANOVA with Dunnett's multiple comparison test. ${ }^{\star} p<0.05,{ }^{\star \star \star} p<0.001,{ }^{\star \star \star \star} p<0.0001$

as proteins were involved in phenazine biosynthesis (phzG1, phzM, phzB1, phzA2), LPS modification ( $\operatorname{arnA}, \operatorname{arnB}, \operatorname{arnD}$, arnT, waaG) and antimicrobial resistance (CprA, PA4775). Type IV pili proteins were enriched in $p m r B$ SNP vs. LESB65 (PilY1, PilF) (Supplementary Data 2). Peptide counts for Cif and Ivy 

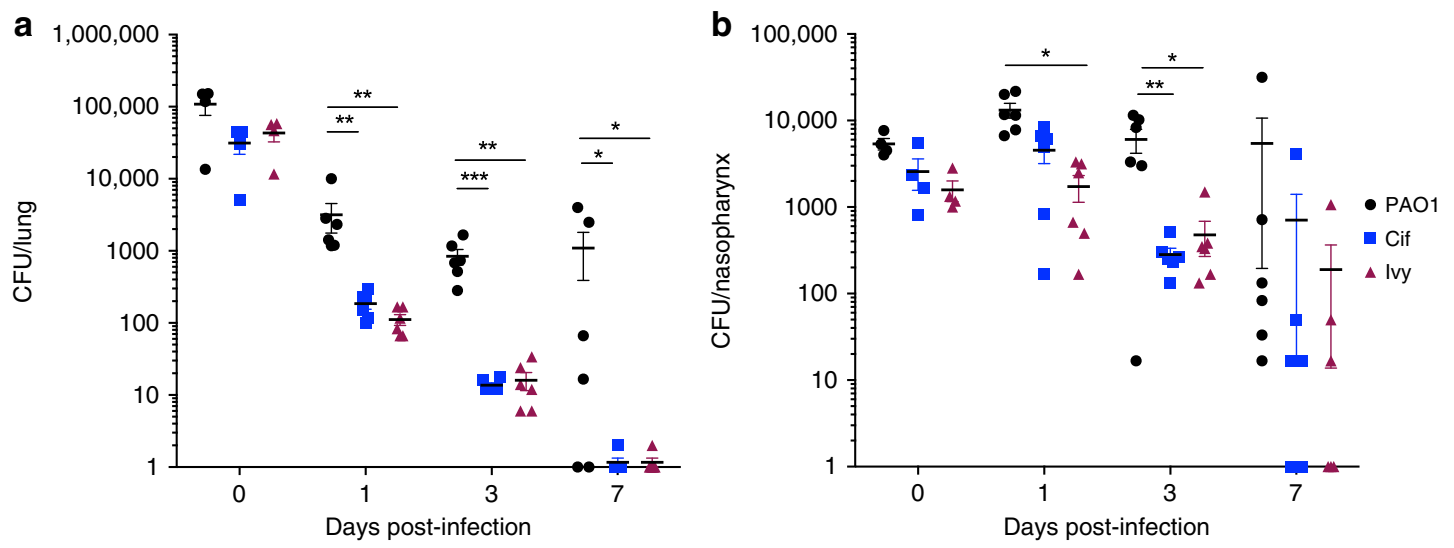

Fig. 4 Cif and Ivy contribute to establishment of lung infection. Mice were infected by intranasal instillation of $1 \times 10^{6}$ colony forming units (CFU) P. aeruginosa PAO1, or PAO1 with transposon insertions in cif or ivy. Mice were culled immediately after infection ( $n=4$ per group) or at 1, 3 and 7 days post-infection ( $n=6$ per group) and CFU in (a) lung and (b) nasopharynx determined by plating of tissue homogenates onto selective agar. Data are from a single experiment. ${ }^{\star} p<0.05,{ }^{\star \star} p<0.01,{ }^{\star \star \star} p<0.001$ in two-way ANOVA with Dunnett's multiple comparison test vs. PAO1

were below the cut-off threshold for analysis in these experiments, but expression differences were confirmed by qPCR (Supplementary Table 3).

Mutations in pmrB in clinical P. aeruginosa isolates. Although loss of $p m r B$ appears to confer phenotypic changes that facilitate establishment of infection in vivo (Figs. 1, 3, 4, 5), it i$s$ also associated with increased susceptibility to antibiotics (Supplementary Fig. 3, Table 1). It is not clear, therefore, whether such mutations would be advantageous in clinical $P$. aeruginosa infection, where intensive antibiotic therapy is the norm. Sequencing of $p m r B$ of 13 Liverpool Epidemic Strain (LES) isolates taken from four CF patients attending the Children's CF centre in Liverpool between 1995 and $2004^{47}$ revealed the presence of SNPs in two isolates from two different individuals. A SNP in isolate 49,194 resulted in conversion of $\mathrm{PmrB}$ arginine 202 to histidine, whilst three SNPs in isolate 49,137 from a different patient led to isoleucine 73 to leucine, glutamate 82 to lysine, and glutamate 225 to glutamine conversions. Both isolates had amino acid changes (at position 202 in 49,194 and 225 in 49,137) in proximity to the conserved H-box region of PmrB. The pmrB SNP isolate from the mouse model that was used in this study and described previously also had an amino acid change in the H-box region (L256Q) ${ }^{6}$. Clinical isolates 49,194 and 49,137 both showed high levels of resistance to lysozyme and were amongst the most susceptible to antibiotics of the isolates from each of the two individuals (Fig. 7 and Supplementary Table 4). Furthermore, both 49,194 and 49,137 showed evidence of increased cif and ivy expression relative to paired isolates from the same patients (Supplementary Table 5). Considerable variation in both lysozyme and antibiotic resistance was observed amongst isolates from a single patient, highlighting the within-patient phenotypic diversity of $P$. aeruginosa (Fig. 7 and Supplementary Table 4). Thus, pmrB mutations are evident in clinical $P$. aeruginosa isolates and are associated with similar phenotypic traits to those identified in the isolates from our mouse model.

\section{Discussion}

Based on our observations, we propose a model whereby pmrB mutants are selected during early infection due to the advantages they confer in colonisation of the host niche (summarised in Fig. 8). Furthermore, as part of a genotypically and phenotypically diverse $P$. aeruginosa population, such as exists in the CF lung ${ }^{48-53}$, pmrB mutation may confer a population-wide benefit through modulation of the host environment via CFTR inhibition. Given the evolutionary trade-offs associated with $\operatorname{pmrB}$ mutation, including enhanced susceptibility to antibiotics, it may be that the maintenance of population diversity is key during clinical infections.

Many of our observations have been made using isolates adapted to the murine lung, which serves as a useful model for human lung infections but does not fully reproduce the features of the CF lung environment. We chose isolate LESB65 for these studies due to its ability to establish long-term infection of the murine respiratory tract ${ }^{6}$. However, as a clinical CF isolate, it might be argued that it is already adapted to the environment of the respiratory tract. However, $P$. aeruginosa infection of the $\mathrm{CF}$ is characterised by genotypic and phenotypic diversity ${ }^{48-53}$, and no single isolate is likely to capture the extent of this diversity. Different mutations are likely to provide fitness benefits at different stages of infection, and pmrB mutation appears most important during early establishment of colonisation. In established infection, other genotypes may take over or coexist in a diverse population. We have demonstrated that $p m r B$ mutations occur amongst clinical isolates (Fig. 8$)^{54}$ and that they have the capacity to make significant contributions to success of infection at the population level. Analysis of a larger pool of clinical isolates from the early stages of infection, to quantify the prevalence of loss-of-function pmrB mutations, and the impact on antimicrobial susceptibility, would provide further insight into this crucial period. Such studies will help determine the extent to which the phenotypes of lysozyme resistance and environmental modulation, we identify here are selected during infection in people with CF.

Collectively, these data highlight the range of $P$. aeruginosa virulence and regulatory mechanisms linked to the $\mathrm{PmrAB}$ two-component regulatory system. Mutations in $p m r B$ may be a sentinel early event in adaptation to the host respiratory tract. The evolutionary trade-off associated with $p m r B$ mutation, whereby increased in vivo fitness is partially offset by antibiotic susceptibility, may offer opportunity for intervention with intensive eradication therapy in early infection. Such an approach would rely on early detection of infection and our earlier findings that the upper respiratory tract acts as an evolutionary nest for $P$. aeruginosa suggests that nasal swabbing could offer a route to earlier diagnosis and treatment in high risk individuals, including those with CF. 

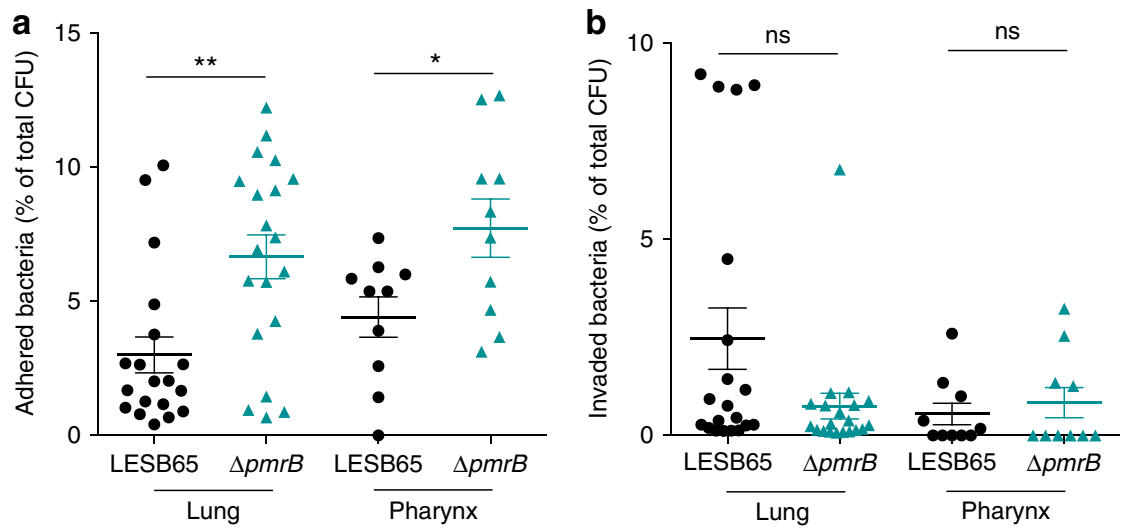

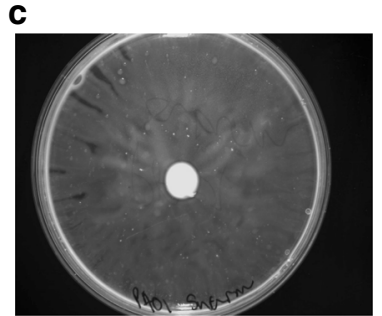

$\mathrm{PAO} 1$

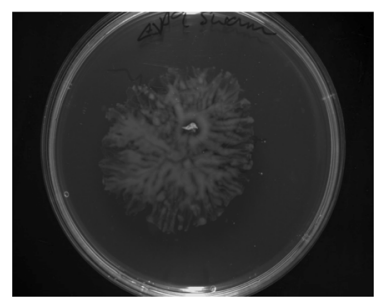

$\Delta p m r B$

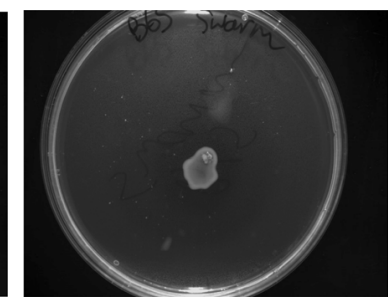

LESB65

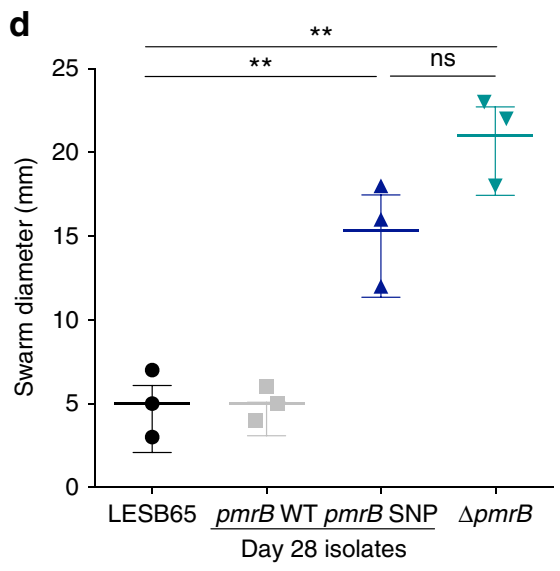

Fig. 5 Pili-dependent processes in LESB65 and $\Delta$ pmrB. a Adhesion and (b) invasion of LESB65 and $\Delta p m r B$ to A549 human alveolar epithelial cells (lung) and human Detroit pharyngeal cells (pharynx). Results are expressed as a percentage of total bacterial numbers per well. Data are a composite of four (lung) or two (pharynx) independent experiments, each containing 5 replicate wells per experimental group. c Swarming motility of PAO1, $\Delta$ pmrB, and LESB65 on semisolid agar. (d) Swarm diameter of isolates grown on semisolid agar. Data points shown are from three independent experiments. ${ }^{\star} p<0.05$, ${ }^{\star \star} p<0.01$ and ns non-significant in unpaired $t$-test with Welch's correction $(\mathbf{a}, \mathbf{b})$ or one-way ANOVA with Tukey post-test (d)

\section{Methods}

Bacterial isolates and gene deletion mutant isolates. LESB65 WT, pmrB WT and $p m r B$ SNP were used and described in a previous study ${ }^{6}$. The LESB65 $\Delta p m r B$ mutant was created specifically for this study. Upstream and downstream regions of $p m r B$ were amplified using Q5 high fidelity DNA polymerase (NEB) using primer sets PmrB1_Xbal 5'-TACTATTCTAGAGGACGACCTGCTGCTCG-3'/ PmrB2_Kpn1 5'AATCCAGGTACCCGGGGGACTCCGGTAGGC-3'-and PmrB3_Kpn1 5'CCAAATGGTACCTAGCCTGCGCATTGGCCGGG-3' PmrB4_EcoRI 5'CCTTGTGAATTCCGGCGAACGCCTGGAGCTA-3'. Restriction sites are underlined. Following digestion with Xbal and Knp1 (upstream regions) or Knpl and EcoRI (downstream region), the amplicons were ligated into the Xbal/EcoRI sites of pEX18Gm "donor" and transformed into Escherichia coli GT115 (selected on gentamicin $10 \mathrm{mg}$ per L). LESB65 WT and a helper E. coli GT115 (pRK2013) were grown on nutrient agar containing $25 \mathrm{mg} / \mathrm{L}$ trimethoprim and $25 \mathrm{mg}$ per L kanamycin respectively. P. aeruginosa LESB 65 was grown at $42^{\circ} \mathrm{C}$ and $E$. coli at $30^{\circ} \mathrm{C}$, overnight. $1 \mathrm{ml}$ of overnight culture was centrifuged at 6500 r.p. $\mathrm{m}$. and resuspended gently in SOB. Recipient, donor, and helper were mixed at a ratio of 3:1:1 ratio and $150 \mu \mathrm{l}$ were pipetted onto the surface of a SOB plate. These were incubated for $20 \mathrm{~h}$ at $30^{\circ} \mathrm{C}$. Bacteria were removed from the plate and reinoculated onto agar plates containing $25 \mathrm{mg}$ per $\mathrm{L}$ trimethoprim and $200 \mathrm{mg}$ per $\mathrm{L}$ gentamicin. Colonies were grown overnight and then negatively selected based on growth on $5 \% 9(\mathrm{v} / \mathrm{v})$ LB Sucrose (with trimethoprim and gentamicin) plates.
Colonies were then grown in LB with trimethoprim $(25 \mathrm{mg}$ per $\mathrm{L})$ and then plated to assess for no gentamicin sensitivity. Knockout mutants were confirmed by Sanger sequencing of the $p m r B$ region (Source Biosciences). PAO1 and transposon mutants from the Two-Allele library were purchased from the Manoil lab (University of Washington) and transposon insertion/gene absence confirmed by PCR (data not shown). Cif and Ivy mutant references are Cif-G08::ISlacZ/hah and PA3902-F10::ISphoA/hah.

Neutrophil degranulation resistance assays. Mouse bone marrow macrophages were isolated from mouse femurs and tibias as previously described ${ }^{55}$. Briefly, a $25-$ gauge needle was used to flush cells from bones and filtered through a $100 \mu \mathrm{m}$ cell strainer. After washing in cold PBS, cells were separated by histopague 1119/1077 density gradient centrifugation. Purity was confirmed by flow cytometry staining for Ly6G and CD11b. HL-60 neutrophils $\left(5 \times 10^{5}\right.$ per $\left.\mathrm{ml}\right)$ or mouse bone marrow macrophages $\left(5 \times 10^{5}\right.$ per $\left.\mathrm{ml}\right)$ were cultured with phorbol myrisate acetate (PMA) for $4 \mathrm{~h}$ to induce degranulation and cell-free culture supernatants were transferred to $1 \times 10^{5}$ pelleted mid-log LESB65, $p m r B$ WT, $p m r B$ SNP and $\triangle p m r B$. CFU were determined after $1 \mathrm{~h}$ exposure, by serial dilution onto LB agar.

Minimum inhibitory concentration. A broth microdilution method was used to determine the MIC of various host antimicrobial peptides and antibiotics on the 
P. aeruginosa strains LESB65, $p m r B$ WT, $p m r B$ SNP and $\triangle p m r B$. Following bacterial growth on $\mathrm{LB}$ agar plates at $37^{\circ} \mathrm{C}$ for $18 \mathrm{~h}$, a single colony was inoculated into $5 \mathrm{ml}$ of LB broth and incubated for a further $18 \mathrm{~h}$ at $37^{\circ} \mathrm{C}$ and 180 r.p.m. A fresh dilution in LB broth was made by incubating $200 \mu$ lof the overnight culture in $5 \mathrm{ml}$ of fresh LB media. A hundred microliters from the fresh bacterial culture were incubated in 96 well-plates with $100 \mu \mathrm{l}$ of 1:2 serially diluted antimicrobial peptide in LB. After a $24 \mathrm{~h}$ static incubation at $37^{\circ} \mathrm{C}$ the $\mathrm{OD}_{600}$ was determined to assess bacterial growth. The antimicrobial peptides used were the cathelicidin antimicrobial peptide LL37, lysozyme from chicken egg white (Sigma-Aldrich, Gillingham, UK), and $\beta$-defensin. The concentration range of the antimicrobial peptide used in the MIC assays varied between peptides: 0-100 $\mu \mathrm{g}$ per $\mathrm{ml}$ for LL37, $0-10 \mu \mathrm{g}$ per $\mathrm{ml}$ for $\beta$-defensin and $0-250 \mu \mathrm{g}$ per $\mathrm{ml}$ for lysozyme.

MIC assay in artificial sputum media. The artificial sputum media was prepared as previously described ${ }^{56,57}$ using DNA from fish sperm (Sigma-Aldrich), mucin from porcine stomach (Sigma-Aldrich), the iron-chelator diethylene triamine pentaacetic acid (Sigma-Aldrich), $\mathrm{NaCl}$ (Sigma-Aldrich), $\mathrm{KCl}$ (Sigma-Aldrich), egg yolk emulsion (Sigma-Aldrich) and all essential and non-essential amino acids (Fisher Scientific, Loughborough, UK and Sigma-Aldrich). Similarly to the MIC assay in LB, the bacterial isolates LESB65, $p m r B$ WT, $p m r B$ SNP and $\triangle p m r B$ were grown in $\mathrm{LB}$ agar plates at $37^{\circ} \mathrm{C}$ for $18 \mathrm{~h}$. Following incubation, a single colony was inoculated into $5 \mathrm{ml}$ of LB and incubated for $18 \mathrm{~h}$ at $37^{\circ} \mathrm{C}$ and 180 r.p.m. The overnight culture was diluted into fresh $\mathrm{LB}$ broth to an $\mathrm{OD}_{600}$ of 0.05 . A 1:100 dilution of the bacterial solution was done in sterile ASM and $1.8 \mathrm{ml}$ of the solution was incubated with $200 \mu \mathrm{l}$ of engineered lysozyme ${ }^{25}$ or tobramycin (Flynn Pharma LTD, Hertfordshire, UK) at different concentrations for $24 \mathrm{~h}$ at $37^{\circ} \mathrm{C}$ and 50 r.p.m.

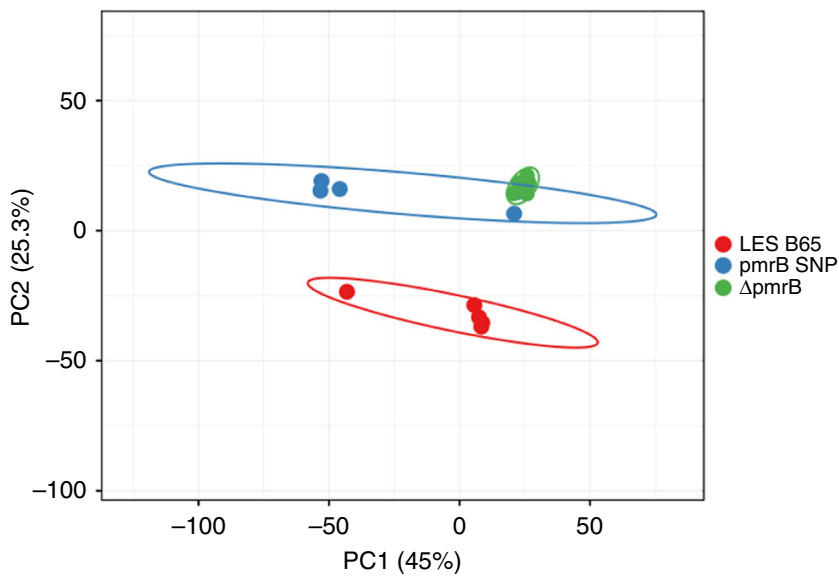

Fig. 6 Principal component biplot of LESB65, pmrB SNP and $\Delta p m r B$. Unit variance scaling is applied to rows; SVD with imputation is used to calculate principal components. $X$ axis and $Y$ axis show principal component 1 and principal component 2 that explain $45 \%$ and $25.3 \%$ of the total variance, respectively. Prediction ellipses are such that with probability 0.95 , a new observation from the same group will fall inside the ellipse. $N=15$ data points
Following incubation, the ASM biofilm was dissolved using Sputasol (Oxoid, Basingstoke, UK). The CFU from each biofilm was determined using the Miles and Misra technique ${ }^{58}$.

Sequencing analysis. Three $P$. aeruginosa LESB65 isolates were sequenced using two Pacific Biosciences RS-II SMRT-cells per isolate (a total of six SMRT-cells) using P2/C4 chemistry: The parental isolate (LESB65), $p m r B$ SNP, and $p m r B$ WT. Subreads were assembled using canu v.1.7.11, and single nucleotide polymorphisms were extracted and annotated using SNIPPY, which takes fake reads generated from the canu consensus sequence, maps these to a reference sequence $(P$. aeruginosa LESB58) using bwa-mem2 and extracts SNP using Freebayes3 and SNPEff4. As Pacific Biosciences sequencing is prone to systematic insertion/deletion errors in homopolymeric tracts, these were removed from the subsequent analysis. Data are available under accessions ERS2269679 (http://ddbj.nig.ac.jp/DRASearch/sample? acc=ERS2269679) (Parent); ERS2269680 (http://ddbj.nig.ac.jp/DRASearch/ sample?acc=ERS2269680) ( $p m r B$ SNP) and ERS2269681 (http://ddbj.nig.ac.jp/ DRASearch/sample?acc $=$ ERS2269681) $($ pmrB WT $)$.

Proteomic analysis. The LESB65, $p m r B$ SNP and $\triangle p m r B$ strains were incubated on LB agar plates for $18 \mathrm{~h}$ at $37^{\circ} \mathrm{C}$. A sweep of colonies were inoculated into $5 \mathrm{ml}$ of $\mathrm{LB}$ broth and incubated for a further $18 \mathrm{~h}$ at $37^{\circ} \mathrm{C}$ and 180 r.p.m. A fresh dilution in LB broth was made by incubating $1.2 \mathrm{ml}$ of the overnight culture into $28.8 \mathrm{ml}$ of fresh LB media. The bacteria were incubated at $37^{\circ} \mathrm{C}$ and 180 r.p.m. to an $\mathrm{OD}_{600}$ between 0.5 and 0.6 and pelleted down at 4500 r.p.m. for $12 \mathrm{~min}$. The pellet was washed once in phosphate buffered saline and stored at $-80^{\circ} \mathrm{C}$ until processing for proteomic analysis. Five replicates per condition were used. Bacterial cell pellets were lysed by sonication $1 \%(\mathrm{w} / \mathrm{v})$ SDC in $50 \mathrm{mM}$ ammonium bicarbonate. Samples were heated at $80^{\circ} \mathrm{C}$ for $15 \mathrm{~min}$ before centrifugation at $12,000 \times g$ to pellet debris. The supernatant was retained, and proteins reduced with $3 \mathrm{mM}$ DTT (Sigma) at $60^{\circ} \mathrm{C}$ for $10 \mathrm{~min}$, cooled, then alkylated with $9 \mathrm{mM}$ iodoacetamide (Sigma) at RT for $30 \mathrm{~min}$ in the dark; all steps were performed with intermittent vortex-mixing. Proteomic-grade trypsin (Sigma) was added at a protein:trypsin ratio of 50:1 and incubated at $37^{\circ} \mathrm{C}$ overnight. SDC was removed by adding TFA to a final concentration of $0.5 \%(\mathrm{v} / \mathrm{v})$. Peptide samples were centrifuged at $12,000 \times g$ for $30 \mathrm{~min}$ to remove precipitated SDC.

NanoLC MS ESI MS/MS analysis. Peptides were analysed by on-line nanoflow LC using the Ultimate 3000 nano system (Dionex/Thermo Fisher Scientific). Samples were loaded onto a trap column (Acclaim PepMap 100, $2 \mathrm{~cm} \times 75 \mu \mathrm{m}$ inner diameter, $\mathrm{C} 18,3 \mu \mathrm{m}, 100 \AA$ ) at $5 \mu \mathrm{lmin}-1$ with an aqueous solution containing $0.1 \%(\mathrm{v} / \mathrm{v})$ TFA and $2 \%(\mathrm{v} / \mathrm{v})$ acetonitrile. After 3 min, the trap column was set in-line an analytical column (Easy-Spray PepMap ${ }^{\circledR}$ RSLC $50 \mathrm{~cm} \times 75 \mu \mathrm{m}$ inner diameter, C18, $2 \mu \mathrm{m}, 100 \AA$ ) fused to a silica nano-electrospray emitter (Dionex). The column was operated at a constant temperature of $35^{\circ} \mathrm{C}$ and the LC system coupled to a Q-Exactive HF mass spectrometer (Thermo Fisher Scientific). Chromatography was performed with a buffer system consisting of $0.1 \%$ formic acid (buffer A) and $80 \%$ acetonitrile in $0.1 \%$ formic acid (buffer B). The peptides were separated by a linear gradient of $3.8-50 \%$ buffer B over $90 \mathrm{~min}$ at a flow rate of $300 \mathrm{nl}$ per min. The Q-Exactive HF was operated in data-dependent mode with survey scans acquired at a resolution of 60,000 . Up to the top 10 most abundant isotope patterns with charge states +2 to +5 from the survey scan were selected with an isolation window of $2.0 \mathrm{Th}$ and fragmented by higher energy collisional dissociation with normalised collision energies of 30 . The maximum ion injection times for the survey scan and the MS/MS scans were 100 and $45 \mathrm{~ms}$, respectively, and the ion target value was set to 3E6 for survey scans and 1E5 for the MS/MS
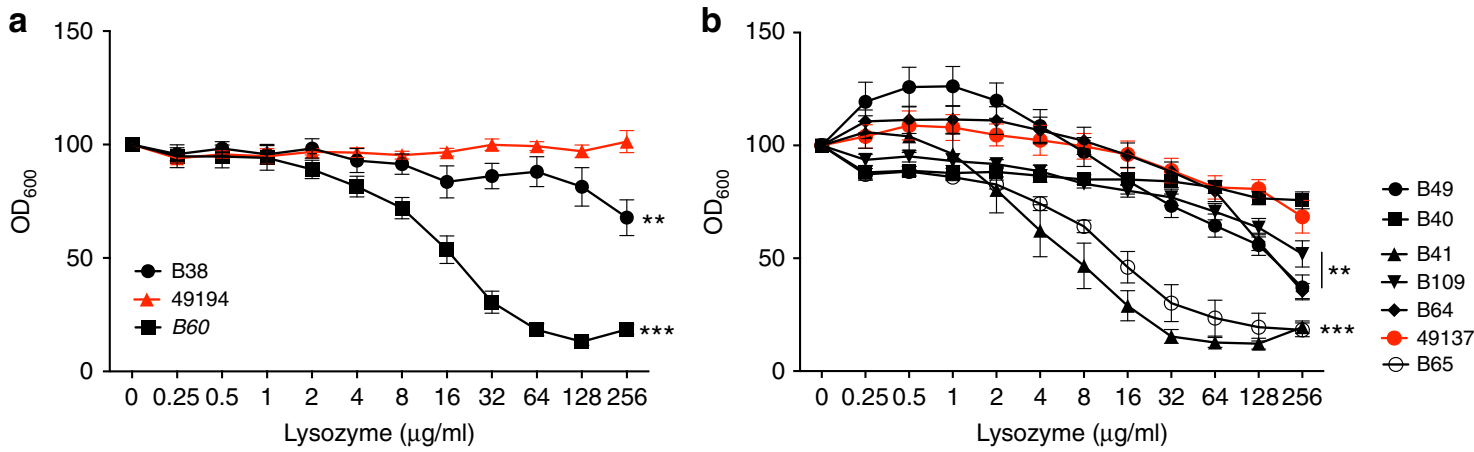

Fig. $7 \mathrm{pmrB}$ mutations are found in patient isolates and are associated with lysozyme resistance. Three isolates from one patient (a) and seven from another (b) were compared for lysozyme resistance. Isolates with at least one pmrB mutation are in red $(49194=\mathrm{R} 202 \mathrm{H}, 49137=173 \mathrm{~L}, \mathrm{E} 82 \mathrm{~K}$, E225Q). $(\mathbf{a}, \mathbf{b})$ Growth in the presence of lysozyme expressed as a percentage of growth in LB $\left(\mathrm{OD}_{600}\right)$. Data are presented as mean $\pm \mathrm{s} . \mathrm{d}$. and are a composite of three independent experiments with each isolate run in triplicate. ${ }^{\star \star} p<0.01,{ }^{\star \star \star} p<0.005$, ns non-significant, vs. 49194 (a) or 49137 (b) in ANOVA analysis performed on $\mathrm{OD}_{600}$ with lysozyme as a covariant 


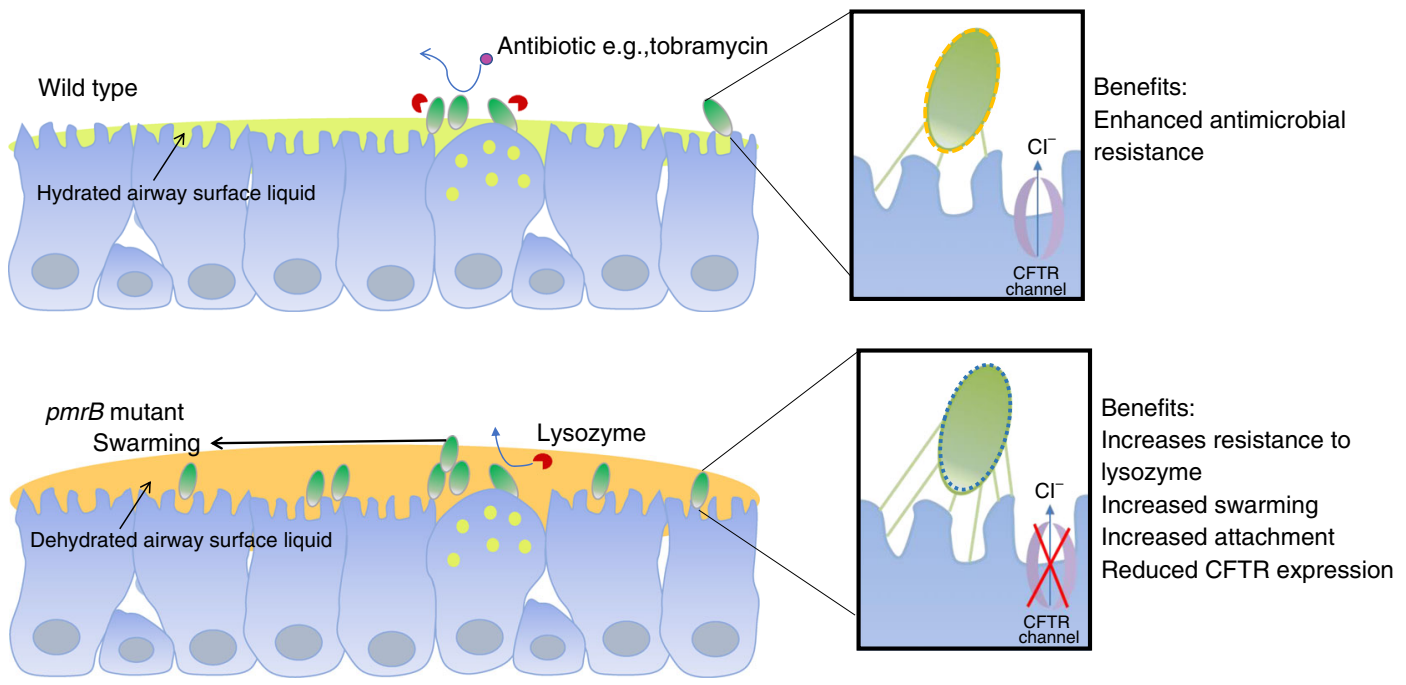

Fig. 8 Proposed mechanism for enhanced infection potential of pmrB mutants. Protein expression changes associated with pmrB mutation may confer an ability to reproduce the phenotypic features of the CF lung via inhibition of host CFTR. Increased expression of type IV pili aids colonisation and adhesion to airway epithelium. Resistance to lysozyme associated with increased ivy production enables persistence in the face of immune pressure. The airway surface liquid is depicted in green for wild type and yellow for the pmrB mutant to denote dehydration resulting from CFTR inhibition

scans. MS/MS events were acquired at a resolution of 30,000. Repetitive sequencing of peptides was minimised through dynamic exclusion of the sequenced peptides for $20 \mathrm{~s}$.

Protein identification and quantification. Thermo RAW files were imported into Progenesis QI for proteomics (version 4.1, Nonlinear Dynamics). Runs were time aligned using default settings and using an auto selected run as reference. Peaks were picked by the software using default settings and filtered to include only peaks with a charge state between +2 and +7 . Spectral data were converted into.mgf files with Progenesis QI for proteomics and exported for peptide identification using the Mascot (version 2.3.02, Matrix Science) search engine. Tandem MS data were searched against translated ORFs from Pseudomonas aeruginosa strain PAO1 (Uniprot reference proteome, UP000002438, December 2016) and a contaminant database (cRAP, GPMDB, 2012) (combined 5733 sequences; 1,909,703 residues). The search parameters were as follows: precursor mass tolerance was set to 10 p.p. $\mathrm{m}$. and fragment mass tolerance was set as $0.05 \mathrm{Da}$. Two missed tryptic cleavages were permitted. Carbamidomethylation (cysteine) was set as a fixed modification and oxidation (methionine) set as variable modification. Mascot search results were further validated using the machine learning algorithm Percolator embedded within Mascot. The Mascot decoy database function was utilised and the false discovery rate was $<1 \%$, while individual percolator ion scores $>13$ indicated identity or extensive homology $(p<0.05)$. Mascot search results were imported into Progenesis QI for proteomics as XML files. Peptide intensities were normalised against the reference run by Progenesis QI for proteomics and these intensities were used to highlight relative differences in protein expression between sample groups. Only proteins with 2 or more identified peptides were included in the dataset. Statistical analysis (ANOVA) of the data was performed using Progenesis QI for proteomics to identify significantly $(p<0.05, q \leq 0.05$, relative fold change $\geq 2$ ) differentially expressed proteins. PCA plots were created using ClustVis using proteins with 2 or greater identified peptides, and $q<0.05$. Unit variance scaling was applied to rows; SVD with imputation was used to calculate principal components.

MALDI-MS of Lipid A modifications. Cultures were initially prepared as described for the proteomic analysis. Bacteria were aliquoted $(1 \mathrm{ml})$ into a $1.5 \mathrm{ml}$ microtube prior to heat-inactivation for $1 \mathrm{~h}$ at $90^{\circ} \mathrm{C}$. The heat-inactivated bacteria were washed three times with $0.5 \mathrm{ml}$ of double distilled water and centrifuged at $9000 \times \mathrm{g}$ for $5 \mathrm{~min}$ and suspended in double distilled water at a final concentration of about $10^{4}$ to $10^{5}$ bacteria per $\mu$ l. Four biological replicates (each with 4 technical replicates) were performed on the LESB65 WT and the $\triangle$ pmrB knockout mutant. Suspensions of $10^{4}$ to $10^{5}$ bacteria per $\mu \mathrm{L}$ were stored at $-80^{\circ} \mathrm{C}$ until required. 0.5 $\mu \mathrm{L}$ of bacterial suspension was spotted onto a stainless steel MALDI plate, $0.5 \mu \mathrm{L}$ of the matrix, 2,5-dihydrobenzoic acid (DHB) made to a final concertation of $10 \mathrm{mg} /$ $\mathrm{mL}$ in a 90:10 chloroform/methanol solution, was spotted on top of the bacterial suspension. MALDI q-TOF analysis were acquired using a Synapt G2-Si (Waters, UK). Negative ion data were recorded in resolution mode, with a mass range of $\mathrm{m} / \mathrm{z}$ 1000-2200. Data was acquired for $180 \mathrm{~s}$ per sample with a scan rate of 1 scan per second and a laser repetition rate of $500 \mathrm{~Hz}$. Average Spectra per acquisition were produced using MassLynx V4.1 (Waters, UK) and processed using the automatic peak detection with background subtraction. Peak intensities were normalised to the average total ion current of the acquisition. A peak was detected at $\mathrm{m} / \mathrm{z}$ meas 1615.9873 corresponds to hexa-acyl Lipid A $(\mathrm{m} / \mathrm{z}$ calc 1615.9879 , $\Delta \mathrm{ppm}-0.37)$. Another peak was detected at $\mathrm{m} / \mathrm{z}$ meas 1445.8531 corresponds to penta-acyl Lipid A $(m / z$ calc $1445.8572, \Delta$ ppm -2.8$)$.

Pyocyanin production. Pyocyanin assays were carried out on culture supernatants during growth in $\mathrm{LB}$ as described previously ${ }^{47}$. The amount of pyocyanin in culture supernatants was quantified by measuring the $\mathrm{OD}_{695}$ value. For overnight cultures, it was important to shake the culture vigorously before obtaining the supernatant This is to ensure that all of the pyocyanin present is in the oxidised form. We used a simple broth test to identify strains with increased blue/green pigment production compared to strain PAO1. Following overnight growth in universals containing $5 \mathrm{ml}$ Luria broth, with shaking at 200 r.p.m. Full pyocyanin production assays were carried out using $250 \mathrm{ml}$ flasks containing $50 \mathrm{ml} \mathrm{LB}$ and absorbance at $695 \mathrm{~nm}$ for supernatants and $600 \mathrm{~nm}$ for cell cultures.

CFTR expression in airway epithelial cells. A549 alveolar epithelial cells (ATCC CCL-185) and Detroit 562 pharyngeal cells (ATCC CCL-138) were obtained from the American Type Culture Collection (Manassas, USA) and epithelial phenotype confirmed by microscopy and flow cytometry. Cells were confirmed mycoplasmafree before performing experiments. Airway cells, at $10^{5}$ per well, were seeded in 12 well plates and grown until the monolayer of cells were confluent, then infected with bacteria $\left(\mathrm{CFU} 10^{4}\right)$ and incubated overnight at $37^{\circ} \mathrm{C}$. Cells were washed once with PBS removed with a cell scraper before four wells from each treatment group were pooled and CFU counts performed. A549 cells were washed and then incubated with anti-CD16/32 (Human TruStain FcX, BioLegend, cat. 422301) in PBS $1 \%(\mathrm{w} / \mathrm{v}) \mathrm{BSA}$ for $30 \mathrm{~min}$ at room temperature. Cells were washed and then fixed and permeabilised (BD Biosciences) before staining with anti-CFTR AF488 (AbCam, cat. ab2784) in permeabilisation buffer (BD Bioscience) for $30 \mathrm{~min}$ at room temperature. Flow cytometry was performed using the BD FACSCelesta (BioSciences, Warrington, United Kingdom) and data analysed using Flow Jo v8.3 (Tree Star Inc, USA). Flow cytometry experiments were performed twice with twothree biological replicates per assay.

Mouse infections. All mouse infection work was performed at the University of Liverpool with prior approval by the UK Home Office and the University of Liverpool Ethics Committee. The female BALB/c mice of 6-8 week old (Charles River, UK) were used for infection experiments and housed in individually ventilated cages for one week to acclimatise prior to infection. Sample size determination was performed as previously described, informed from previous work comparing $P$. aeruginosa infection density in mice ${ }^{6}$. Mice were randomly assigned to a cage (experimental group) on arrival at the unit by staff with no role in study design. $2 \times 10^{6}$ colony forming units of PAO1 or PAO1 transposon mutants were instilled into the nares of mice that had been lightly anaesthetised with a mixture of isoflurane and oxygen. Cage labels were reversed to ensure researchers were blinded to experimental groups. Mice were monitored for signs of disease and culled at pre-determined time points. Nasopharyngeal tissue and lungs were removed post-mortem and homogenised in $3 \mathrm{ml}$ PBS using an IKA T10 handheld tissue homogeniser (IKA, USA), before serial dilution onto Pseudomonas selective agar (Oxoid, UK) for enumeration of infectious burden. Following enumeration, researchers were unblinded. No animals were excluded from analysis. 
Adhesion and invasion of airway epithelial cells. Differential adhesion and invasion assay performed as previously described ${ }^{59,60}$. Briefly, A549 alveolar epithelial cells (ATCC CCL-185) or Detroit 562 pharyngeal cells (ATCC CCL-138) (both at $10^{5}$ per well) were seeded in $2 \times 24$ well plates and grown until confluent then infected with bacteria (CFU $\left.10^{4}\right)$ and incubated for two hours. Cells were washed five times with PBS to remove non-adherent bacteria and for invasion assay, samples were incubated for a further two hours in media with $600 \mathrm{mg}$ per $\mathrm{ml}$ gentamicin to kill extracellular and adherent bacteria (adhesion control was performed without gentamicin). The cells were washed three times with PBS and 100 $\mu \mathrm{l}$ trypsin added to remove cells from the plate (incubated for $10 \mathrm{~min}$ ), then cells lysed with $100 \mu \mathrm{l} 0.05 \%$ (v/v) Triton x100 in incubator for $10 \mathrm{~min}$. Samples were plated on LB agar in 10-fold dilutions up to $10^{-6}$ using the Miles \& Misra technique ${ }^{58}$ and incubated at $37^{\circ} \mathrm{C}$ for $48 \mathrm{~h}$, at which point bacterial CFU were counted. Experimental conditions were performed in duplicate and repeated five times. Adhesion CFU per ml was calculated by removing invasion counts from invasion plus adhesion counts.

Bacterial motility assays. Swim and swarm petri dish plates were inoculated on the surface as described previously ${ }^{61,62}$, then incubated for 14 and $16 \mathrm{~h}$, respectively, at $37^{\circ} \mathrm{C}$. Swim plates were first loosely wrapped in cling film to prevent dehydration, and incubated without inversion. The visible diameter of bacterial growth was measured at the widest point after incubation. Twitching motility was determined by subsurface stab assay of a colony of bacteria deep into LB agar until it touched the agar-dish interface ${ }^{63}$, then motility zone measured after incubation for $24 \mathrm{~h}$ at $37^{\circ} \mathrm{C}$. To visualise the twitching motility diameter, agar was removed with tweezers and plates stained with $10 \mathrm{ml} 0.25 \%(\mathrm{w} / \mathrm{v})$ crystal violet for $30 \mathrm{~min}$ before rinsing with water. An isolate with diameter $<10 \mathrm{~mm}$ at widest point was considered twitching motility impaired, with experiments repeated five times.

PCR amplification and sequencing of pmrB. PCR amplification was carried out as described previously ${ }^{64}$. Amplification of the $p m r B$ region was carried out using the oligonucleotide primer sets pmrBF (5' - CCTACCCCTCTCGCTGAAG- $\left.3^{\prime}\right) / \mathrm{pmrBR}$ (5'-GATGTTCATCCGGGTCTCCT-3'). PCR amplicons were purified using Qiaquick PCR purification columns (Qiagen) and sequenced by Source Bioscience using the same oligonucleotide primers employed in the PCR amplification.

Quantitative real-time PCR (qPCR) and analysis. qPCR was performed using the Rotor-Gene Q cycler (Qiagen) as described previously ${ }^{6}$. Specific forward and reverse primers were used to target cif (cif_F-TGGCACTTCAGTTTCTTCGC and cif_R-TGTTGCTGGAATGGGACTTG primerS) and ivy (ivy_F-GAGTATCCAG ACTGCTCTCCC and ivy_R-TGGGGTTTGCAACTGTTGG primers). Each qPCR was performed with three biological replicates and in duplicate on each run. A standard curve of known copy number was performed in each run. Obtained data were analysed using the Rotor-Gene Q series software (Qiagen). Relative expression levels of genes cif and ivy were calculated using the two-standard curve method. Genes proC and $r p o D$, encoding the pyrroline-5-carbocylate reductase and sigma factor RpoD, respectively, were used as reference genes. A standard curve was constructed for each target and reference genes.

Statistical analysis. Statistical analysis was performed in GraphPad Prism V 6.0 (GraphPad Software, La Jolla, USA) and data were tested for normality and to define the variance of each group tested. All multi-parameter analyses included corrections for multiple comparisons and data are presented as mean \pm standard deviation (SD) unless otherwise stated. For MIC determination, median values are stated and odd numbers of replicates performed to avoid derived values.

Data availability. PacBio sequence data are available from DDBJ sequence read archive under accessions ERS2269679 (LESB65), ERS2269680 (pmrB SNP) and ERS2269681 ( $p m r B$ WT). The mass spectrometry proteomics data have been deposited to the ProteomeXchange Consortium via the PRIDE ${ }^{65}$ partner repository with the dataset identifiers PXD009705 and 10.6019/PXD009705. All other data supporting the findings of the study are available in this article and its Supplementary Information files, or from the corresponding authors on request.

Received: 2 October 2017 Accepted: 6 June 2018

Published online: 06 July 2018

\section{References}

1. Emerson, J., Rosenfeld, M., McNamara, S., Ramsey, B. \& Gibson, R. L. Pseudomonas aeruginosa and other predictors of mortality and morbidity in young children with cystic fibrosis. Pediatr. Pulmonol. 34, 91-100 (2002).

2. Gibson, R. L., Burns, J. L. \& Ramsey, B. W. Pathophysiology and management of pulmonary infections in cystic fibrosis. Am. J. Respir. Crit. Care. Med. 168, 918-951 (2003).
3. Selezska, K. et al. Pseudomonas aeruginosa population structure revisited under environmental focus: impact of water quality and phage pressure. Environ. Microbiol. 14, 1952-1967 (2012).

4. Shen, K. et al. Extensive genomic plasticity in Pseudomonas aeruginosa revealed by identification and distribution studies of novel genes among clinical isolates. Infect. Immun. 74, 5272-5283 (2006).

5. Aanaes, K. et al. Secretory IgA as a diagnostic tool for Pseudomonas aeruginosa respiratory colonization. J. Cyst. Fibros. 12, 81-87 (2013).

6. Fothergill, J. L., Neill, D. R., Loman, N., Winstanley, C. \& Kadioglu, A. Pseudomonas aeruginosa adaptation in the nasopharyngeal reservoir leads to migration and persistence in the lungs. Nat. Commun. 5, 4780 (2014).

7. Hansen, S. K. et al. Evolution and diversification of Pseudomonas aeruginosa in the paranasal sinuses of cystic fibrosis children have implications for chronic lung infection. ISME J. 6, 31-45 (2012).

8. Johansen, H. K. et al. Colonisation and infection of the paranasal sinuses in cystic fibrosis patients is accompanied by a reduced PMN response. J. Cyst. Fibros. 11, 525-531 (2012).

9. Winstanley, C. et al. Newly introduced genomic prophage islands are critical determinants of in vivo competitiveness in the Liverpool Epidemic Strain of Pseudomonas aeruginosa. Genome Res. 19, 12-23 (2009).

10. Cullen, L. et al. Phenotypic characterization of an international Pseudomonas aeruginosa reference panel: strains of cystic fibrosis (CF) origin show less in vivo virulence than non-CF strains. Microbiology 161, 1961-1977 (2015).

11. Gooderham, W. J. et al. The sensor kinase PhoQ mediates virulence in Pseudomonas aeruginosa. Microbiology 155, 699-711 (2009).

12. Furukawa, S., Kuchma, S. L. \& O’Toole, G. A. Keeping their options open: acute versus persistent infections. J. Bacteriol. 188, 1211-1217 (2006).

13. Lapouge, K., Schubert, M., Allain, F. H. \& Haas, D. Gac/Rsm signal transduction pathway of gamma-proteobacteria: from RNA recognition to regulation of social behaviour. Mol. Microbiol. 67, 241-253 (2008).

14. Moskowitz, S. M., Ernst, R. K. \& Miller, S. I. PmrAB, a two-component regulatory system of Pseudomonas aeruginosa that modulates resistance to cationic antimicrobial peptides and addition of aminoarabinose to lipid A. J. Bacteriol. 186, 575-579 (2004).

15. Ganz, T. \& Weiss, J. Antimicrobial peptides of phagocytes and epithelia. Semin. Hematol. 34, 343-354 (1997).

16. Mason, D. Y. \& Taylor, C. R. The distribution of muramidase (lysozyme) in human tissues. J. Clin. Pathol. 28, 124-132 (1975).

17. Ellison, R. T. 3rd \& Giehl, T. J. Killing of gram-negative bacteria by lactoferrin and lysozyme. J. Clin. Invest. 88, 1080-1091 (1991).

18. Nash, J. A., Ballard, T. N., Weaver, T. E. \& Akinbi, H. T. The peptidoglycandegrading property of lysozyme is not required for bactericidal activity in vivo. J. Immunol. 177, 519-526 (2006).

19. Jenzano, J. W., Hogan, S. L. \& Lundblad, R. L. Factors influencing measurement of human salivary lysozyme in lysoplate and turbidimetric assays. J. Clin. Microbiol. 24, 963-967 (1986).

20. Jochumsen, N. et al. The evolution of antimicrobial peptide resistance in Pseudomonas aeruginosa is shaped by strong epistatic interactions. Nat. Commun. 7, 13002 (2016)

21. Barbosa, C. et al. Alternative evolutionary paths to bacterial antibiotic resistance cause distinct collateral effects. Mol. Biol. Evol. 34, 2229-2244 (2017).

22. Hoiby, N., Ciofu, O. \& Bjarnsholt, T. Pseudomonas aeruginosa biofilms in cystic fibrosis. Future Microbiol. 5, 1663-1674 (2010).

23. Davies, J. C. \& Bilton, D. Bugs, biofilms, and resistance in cystic fibrosis. Respir. Care 54, 628-640 (2009).

24. Griswold, K. E. et al. Bioengineered lysozyme in combination therapies for Pseudomonas aeruginosa lung infections. Bioengineered 5, 143-147 (2014)

25. Scanlon, T. C. et al. Enhanced antimicrobial activity of engineered human lysozyme. ACS Chem. Biol. 5, 809-818 (2010).

26. Gill, A., Scanlon, T. C., Osipovitch, D. C., Madden, D. R. \& Griswold, K. E. Crystal structure of a charge engineered human lysozyme having enhanced bactericidal activity. PLoS ONE 6, el6788 (2011).

27. Macfarlane, E. L., Kwasnicka, A. \& Hancock, R. E. Role of Pseudomonas aeruginosa PhoP-phoQ in resistance to antimicrobial cationic peptides and aminoglycosides. Microbiology 146 (Pt 10), 2543-2554 (2000).

28. McPhee, J. B., Lewenza, S. \& Hancock, R. E. Cationic antimicrobial peptides activate a two-component regulatory system, $\mathrm{PmrA}-\mathrm{PmrB}$, that regulates resistance to polymyxin $B$ and cationic antimicrobial peptides in Pseudomonas aeruginosa. Mol. Microbiol. 50, 205-217 (2003).

29. Johnson, L., Mulcahy, H., Kanevets, U., Shi, Y. \& Lewenza, S. Surface-localized spermidine protects the Pseudomonas aeruginosa outer membrane from antibiotic treatment and oxidative stress. J. Bacteriol. 194, 813-826 (2012).

30. Gutu, A. D., Rodgers, N. S., Park, J. \& Moskowitz, S. M. Pseudomonas aeruginosa high-level resistance to polymyxins and other antimicrobial peptides requires cprA, a gene that is disrupted in the PAO1 strain. Antimicrob. Agents Chemother. 59, 5377-5387 (2015). 
31. Kohler, T., Epp, S. F., Curty, L. K. \& Pechere, J. C. Characterization of MexT, the regulator of the MexE-MexF-OprN multidrug efflux system of Pseudomonas aeruginosa. J. Bacteriol. 181, 6300-6305 (1999).

32. Denning, G. M. et al. Pseudomonas pyocyanin increases interleukin-8 expression by human airway epithelial cells. Infect. Immun. 66, 5777-5784 (1998).

33. Winstanley, C. \& Fothergill, J. L. The role of quorum sensing in chronic cystic fibrosis Pseudomonas aeruginosa infections. FEMS Microbiol. Lett. 290, 1-9 (2009).

34. Monchois, V., Abergel, C., Sturgis, J., Jeudy, S. \& Claverie, J. M. Escherichia coli ykfE ORFan gene encodes a potent inhibitor of C-type lysozyme. J. Biol. Chem. 276, 18437-18441 (2001).

35. Deckers, D., Vanlint, D., Callewaert, L., Aertsen, A. \& Michiels, C. W. Role of the lysozyme inhibitor Ivy in growth or survival of Escherichia coli and Pseudomonas aeruginosa bacteria in hen egg white and in human saliva and breast milk. Appl. Environ. Microbiol. 74, 4434-4439 (2008).

36. Callewaert, L. et al. A new family of lysozyme inhibitors contributing to lysozyme tolerance in gram-negative bacteria. PLoS Pathog. 4, e1000019 (2008).

37. Swiatecka-Urban, A. et al. Pseudomonas aeruginosa inhibits endocytic recycling of CFTR in polarized human airway epithelial cells. Am. J. Physiol. Cell. Physiol. 290, C862-C872 (2006).

38. MacEachran, D. P. et al. The Pseudomonas aeruginosa secreted protein PA2934 decreases apical membrane expression of the cystic fibrosis transmembrane conductance regulator. Infect. Immun. 75, 3902-3912 (2007).

39. Persat, A., Inclan, Y. F., Engel, J. N., Stone, H. A. \& Gitai, Z. Type IV pili mechanochemically regulate virulence factors in Pseudomonas aeruginosa. Proc. Natl Acad. Sci. USA 112, 7563-7568 (2015).

40. Mann, E. E. \& Wozniak, D. J. Pseudomonas biofilm matrix composition and niche biology. FEMS Microbiol. Rev. 36, 893-916 (2012).

41. Pier, G. B., Grout, M. \& Zaidi, T. S. Cystic fibrosis transmembrane conductance regulator is an epithelial cell receptor for clearance of Pseudomonas aeruginosa from the lung. Proc. Natl Acad. Sci. USA 94, 12088-12093 (1997)

42. Pier, G. B., Grout, M., Zaidi, T. S. \& Goldberg, J. B. How mutant CFTR may contribute to Pseudomonas aeruginosa infection in cystic fibrosis. Am. J. Respir. Crit. Care Med. 154, S175-S182 (1996).

43. Pier, G. B. et al. Role of mutant CFTR in hypersusceptibility of cystic fibrosis patients to lung infections. Science 271, 64-67 (1996).

44. Conrad, J. C. et al. Flagella and pili-mediated near-surface single-cell motility mechanisms in P. aeruginosa. Biophys. J. 100, 1608-1616 (2011).

45. Kohler, T., Curty, L. K., Barja, F., van Delden, C. \& Pechere, J. C. Swarming of Pseudomonas aeruginosa is dependent on cell-to-cell signaling and requires flagella and pili. J. Bacteriol. 182, 5990-5996 (2000).

46. Overhage, J., Bains, M., Brazas, M. D. \& Hancock, R. E. Swarming of Pseudomonas aeruginosa is a complex adaptation leading to increased production of virulence factors and antibiotic resistance. J. Bacteriol. 190 2671-2679 (2008).

47. Fothergill, J. L. et al. Widespread pyocyanin over-production among isolates of a cystic fibrosis epidemic strain. BMC Microbiol. 7, 45 (2007).

48. Clark, S. T. et al. Phenotypic diversity within a Pseudomonas aeruginosa population infecting an adult with cystic fibrosis. Sci. Rep. 5, 10932 (2015).

49. Fothergill, J. L., Mowat, E., Ledson, M. J., Walshaw, M. J. \& Winstanley, C. Fluctuations in phenotypes and genotypes within populations of Pseudomonas aeruginosa in the cystic fibrosis lung during pulmonary exacerbations. J. Med. Microbiol. 59, 472-481 (2010).

50. Wilder, C. N., Allada, G. \& Schuster, M. Instantaneous within-patient diversity of Pseudomonas aeruginosa quorum-sensing populations from cystic fibrosis lung infections. Infect. Immun. 77, 5631-5639 (2009).

51. Williams, D. et al. Divergent, coexisting Pseudomonas aeruginosa lineages in chronic cystic fibrosis lung infections. Am. J. Respir. Crit. Care Med. 191, 775-785 (2015).

52. Workentine, M. L. et al. Phenotypic heterogeneity of Pseudomonas aeruginosa populations in a cystic fibrosis patient. PLoS One 8, e60225 (2013).

53. Winstanley, C., O’Brien, S. \& Brockhurst, M. A. Pseudomonas aeruginosa evolutionary adaptation and diversification in cystic fibrosis chronic lung infections. Trends Microbiol. 24, 327-337 (2016).

54. Yang, L. et al. Evolutionary dynamics of bacteria in a human host environment. Proc. Natl Acad. Sci. USA 108, 7481-7486 (2011).

55. Swamydas, M., Luo, Y., Dorf, M. E. \& Lionakis, M. S. Isolation of Mouse Neutrophils. Curr. Protoc. Immunol. 110, 20 21-23 2015 (2015).

56. Sriramulu, D. D., Lunsdorf, H., Lam, J. S. \& Romling, U. Microcolony formation: a novel biofilm model of Pseudomonas aeruginosa for the cystic fibrosis lung. J. Med. Microbiol. 54, 667-676 (2005).

57. Kirchner, S. et al. Use of artificial sputum medium to test antibiotic efficacy against Pseudomonas aeruginosa in conditions more relevant to the cystic fibrosis lung. J. Vis. Exp. 64, e3857 (2012).
58. Miles, A. A., Misra, S. S. \& Irwin, J. O. The estimation of the bactericidal power of the blood. J. Hyg. 38, 732-749 (1938).

59. Nyberg, P., Rasmussen, M., Von Pawel-Rammingen, U. \& Bjorck, L. SpeB modulates fibronectin-dependent internalization of Streptococcus pyogenes by efficient proteolysis of cell-wall-anchored protein F1. Microbiology 150, 1559-1569 (2004).

60. Berry, A. M. \& Paton, J. C. Sequence heterogeneity of PsaA, a 37-kilodalton putative adhesin essential for virulence of Streptococcus pneumoniae. Infect. Immun. 64, 5255-5262 (1996).

61. Ha, D. G., Kuchma, S. L. \& O’Toole, G. A. Plate-based assay for swarming motility in Pseudomonas aeruginosa. Methods Mol. Biol. 1149, 67-72 (2014).

62. Tremblay, J. \& Deziel, E. Improving the reproducibility of Pseudomonas aeruginosa swarming motility assays. J. Basic Microbiol. 48, 509-515 (2008).

63. Turnbull, L. \& Whitchurch, C. B. Motility assay: twitching motility. Methods Mol. Biol. 1149, 73-86 (2014).

64. Parsons, Y. N. et al. Use of subtractive hybridization to identify a diagnostic probe for a cystic fibrosis epidemic strain of Pseudomonas aeruginosa. J. Clin. Microbiol. 40, 4607-4611 (2002).

65. Vizcaino, J. A. et al. 2016 update of the PRIDE database and related tools. Nucleic Acids Res. 44, 447-456 (2016).

\section{Acknowledgements}

The study was funded by a British Lung Foundation grant awarded to J.F. and D.N., an Action Medical Research project grant awarded to J.F., D.N., C.W. and A.K. and an MRC project grant awarded to A.K. D.N. is supported by a Sir Henry Dale Fellowship jointly funded by the Wellcome Trust and the Royal Society (Grant Number 204457/Z/16/Z). J.F. is supported by a Medical Research Foundation Fellowship. K.G. and Y.F. were supported by the National Institutes of Health (Grant Number NIH P20GM113132). S.A. is affiliated to the National Institute for Health Research Health Protection Research Unit (NIHR HPRU) in Emerging and Zoonotic Infections at University of Liverpool in partnership with Public Health England (PHE), in collaboration with Liverpool School of Tropical Medicine. We are grateful to the Centre of Proteome Research, supported by the technology directorate, University of Liverpool, and particularly Dr P. Brownridge for instrumentation support. We acknowledge the Manoil lab and NIH grant P30 DK089507 for provision of PAO1 transposon mutants.

\section{Author contributions}

C.W., J.F., A.K. and D.N. conceived, designed and supervised the study and contributed equally throughout. L.B.M., V.S., I.G., J.W., E.W., S.A., J.S., S.P., J.D., A.C., J.F. and D.N. performed experiments. Y.F. and K.G. provided reagents. I.G., S.A., J.S., C.W., A.K., J.F. and D.N. analysed data. J.F. and D.N. wrote the paper with input from all authors. The views expressed are those of the author(s) and not necessarily those of the NHS, the NIHR, the Department of Health or Public Health England.

\section{Additional information}

Supplementary Information accompanies this paper at https://doi.org/10.1038/s41467018-04996-x.

\section{Competing interests: The authors declare no competing interests.}

Reprints and permission information is available online at http://npg.nature.com/ reprintsandpermissions/

Publisher's note: Springer Nature remains neutral with regard to jurisdictional claims in published maps and institutional affiliations.

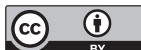

Open Access This article is licensed under a Creative Commons Attribution 4.0 International License, which permits use, sharing, adaptation, distribution and reproduction in any medium or format, as long as you give appropriate credit to the original author(s) and the source, provide a link to the Creative Commons license, and indicate if changes were made. The images or other third party material in this article are included in the article's Creative Commons license, unless indicated otherwise in a credit line to the material. If material is not included in the article's Creative Commons license and your intended use is not permitted by statutory regulation or exceeds the permitted use, you will need to obtain permission directly from the copyright holder. To view a copy of this license, visit http://creativecommons.org/ licenses/by/4.0/.

(C) The Author(s) 2018 